posts in 1 year (UC: 68.6 [47.5] vs CD: 42.2 [29.6], $p=0.12$ ) or in the number of words per post (UC: 449 [149] vs CD: 475 [263], $p=0.76$ ) between groups. Overall, there was no difference in the mean [SD] readability (FRES) scores (UC: 71 [7.0] vs CD: 67.9 [11.0], $p=0.39$ ): reportedly, easily understood by students aged between 13 and 14 years. No differences were seen in the median number of comments or links per post between groups. Overall, the majority of posts detailed personal experiences of IBD, with no differences between groups (UC: 51\% [326/642] CD: 53\% [264/499], $\mathrm{p}=0.51$ ). Active disease was more frequently coded as a precipitant reason for making an entry in UC 25\% [159/642] vs CD 17\% [84/499], $\mathrm{p}=0.001$. Patients with CD more frequently made entries in order to offer IBD related advice, including recent research advances, than patients with UC (UC: 4\% [26/649] vs CD: 24\% [118/499], $\mathrm{p}<0.0001)$ : whereas patients with UC were more likely to use entries for non-IBD related social networking (UC: $24 \%$ 153/642 vs CD 14\% [72/499], $\mathrm{p}<0.0001)$. In total, only $6 \%(63 / 1141)$ of posts referred to psychological distress, with no differences between groups.

Conclusion The content of blogs differs according to disease type, but relatively few bloggers refer to psychological distress in their posts. Online platforms, such as a blogs, may however be a new way of providing patients with IBD psychological support.

Competing interests None declared.

\section{OC-160 TELEMEDICINE SYSTEMS IN IBD MANAGEMENT-ARE PATIENTS READY?}

doi:10.1136/gutjnl-2012-302514a.160

J Landy, ${ }^{*}$ S T Peake, A Akbar, A L Hart. IBD Unit, St Mark's Hospital, London, UK

Introduction Inflammatory bowel disease (IBD) is a chronic condition characterised by periods of exacerbations and remission, requiring regular medical follow-up. IBD frequently affects patients of working age and the need for life-long follow-up can have significant personal and societal economic implications. Telemedicine systems, could reduce this burden and have been shown to be successful and highly accepted by patients with other chronic diseases. ${ }^{1}$ We aimed to assess the potential technologies by which telemedicine might be employed in our population of IBD patients and the level of patients' acceptance of telemedicine systems in their management.

Methods Patients attending the specialist IBD outpatient clinic were surveyed over a 6-week period. Demographic data, access to technology and acceptance of telemedical systems for the management of their disease were assessed.

Results 52 IBD patients (48\%) responded and completed the survey. $52 \%$ had a diagnosis of UC; $48 \%$ Crohn's disease. $58 \%$ were female. $85 \%$ of patients were aged $18-65$ years and English was the first language in $86 \% .94 \%$ of patients had home access to the internet. $56 \%$ owned smartphones and $52 \%$ used apps regularly. $46 \%$ of patients regularly used web video calling. $85 \%$ of patients wanted electronic access to their personal health data. $65 \%$ and $54 \%$ of patients preferred text or email respectively, to be used for reminders of disease monitoring investigations. $65 \%$ would choose telephone follow-up, while only 38\% would select web based follow-up. $42 \%$ of patients indicated they would undertake web supported self-management of their IBD.

Conclusion IBD patients are of working age and have access to web based and smartphone technologies that could be used in the management of their IBD. IBD patients desire e-health access and the use of technology for communication regarding their disease management as well as web-based monitoring and self management of their IBD. Patients with IBD are ready for telemedicine systems to be employed as an adjunct in the management of their disease.

\section{Competing interests None declared.}

\section{REFERENCE}

1. Mclean S, Protti D, Sheikh A. Tele healthcare for longterm conditions. BMJ 2011;342:d120.

\section{Inflammatory bowel disease free papers OC-161 INVESTIGATION OF THE ACTION OF HISTONE DEACETYLASE INHIBITORS IN EX VIVO AND IN VITRO MODELS OF INFLAMMATORY BOWEL DISEASE}

doi:10.1136/gutjnl-2012-302514a.161

${ }^{1} \mathrm{~A} J \mathrm{P}$ Edwards, ${ }^{*}{ }^{1} \mathrm{M}$ Gwiggner, ${ }^{1} \mathrm{~A} \mathrm{G}$ Claridge, ${ }^{1} \mathrm{R} \mathrm{J}$ Morgan-Walsh, ${ }^{2} \mathrm{~A} L$ Hayden, ${ }^{3} \mathrm{~A}$ Bateman, ${ }^{4} \mathrm{~J}$ R F Cummings, ${ }^{2} \mathrm{G}$ K Packham, ${ }^{1} \mathrm{~S}$ L F Pender. ${ }^{1}$ Department of Clinical and Experimental Sciences, Faculty of Medicine, University of Southampton, Southampton, UK; ${ }^{2}$ Department of Cancer Sciences, University of Southampton, Southampton, UK; ${ }^{3}$ Department of Pathology, University Hospital Southampton NHS Foundation Trust, Southampton, UK; ${ }^{4}$ Department of Gastroenterology, University Hospital Southampton NHS Foundation Trust, Southampton, UK

Introduction The management of inflammatory bowel disease (IBD) has evolved rapidly with anti-TNF $\alpha$ agents and the more appropriate use of immunomodulators. Despite this, a large therapeutic gap remains to be filled. Histone deacetylase (HDAC) inhibitors (HDI) preserve acetylation of core histones which prevents chromatin from condensing thus facilitating gene transcription. HDI have been approved for the treatment of sub-cutaneous T-cell lymphoma and recent work has shown that HDI may have a protective antiinflammatory effect in murine models of UC (Tao et al 2007). We hypothesise that HDI have anti-inflammatory effects in human IBD and aim to investigate this using a human ex vivo model of IBD.

Methods Carefully phenotyped patients with active UC $(n=7)$ and $\mathrm{CD}(\mathrm{n}=10)$ undergoing lower GI endoscopy had 8 pinch biopsies $\left(\approx 3 \mathrm{~mm}^{2}\right)$ taken for these experiments. Biopsies were cultured ex vivo at an air liquid interface for $8 \mathrm{~h} \pm \mathrm{FK} 228$ or SAHA. Additionally, gut fibroblasts isolated from resection tissue were used to model mucosal plasticity in vitro \pm TNF- $\alpha$ and FK228 and a monocytic cell line (U937) was differentiated in vitro with GMCSF \pm FK228. Supernatants, RNA and tissue were collected for analysis by qRTPCR, mesoscale assay, western blotting and histology.

Results Nanomolar levels of FK228 significantly reduce mRNA expression and protein secretion of Th1 and Th2 cytokines and proinflammatory mediators (IL-8, MMP-1, $-3,-9$ and -12 ) in the ex vivo UC and CD biopsy models. Data suggest that FK228 can decrease mRNA expression of Th1 and Th17 signalling molecules in the ex vivo models. FK228 significantly decreases IL-8, MMP-3 and MMP12 mRNA expression as well as IL-8 and MMP-3 protein secretion by gut fibroblasts and decreases GMCSF induced MMP-12 protein production by U937 cells.

Conclusion This human ex vivo biopsy culture model is relevant for pre-clinical study of drug action in IBD as it utilises small amounts of tissue in a human system. FK228 produces significant antiinflammatory effects at low doses by reducing cytokines and MMPs in this model. FK228 is able to act upon specific cells found in the gut such as lymphocytes, fibroblasts and monocytes to elicit antiinflammatory effects. Based upon our initial findings, further investigation of the role of FK228 in IBD is warranted.

Competing interests None declared.

\section{OC-162 THE ROLE OF MICRORNAS MIR-31 AND MIR-155 IN THE DEREGULATION OF THE IL-13 PATHWAY IN ULCERATIVE COLITIS}

doi:10.1136/gutjnl-2012-302514a.162

${ }^{1} \mathrm{M}$ Gwiggner, ${ }^{*} \mathrm{~A}$ Claridge, ${ }^{2} \mathrm{~F}$ Cummings, ${ }^{1} \mathrm{~T}$ Sanchez-Elsner. ${ }^{1}$ Department of Clinical and Experimental Sciences, University of Southampton, Southampton General Hospital, 
Southampton, UK; ${ }^{2}$ Department of Gastroenterology, University Hospital Southampton NHS Trust, Southampton, UK

Introduction Ulcerative colitis (UC) is an inflammatory disease of the colonic mucosa driven by a Th2-like response in which IL-13 leads to toxic effects to the mucosa and activation of the IL-13/STAT6 pathways. Inhibition of STAT6 activation has been shown to reduce the inflammatory response in UC. MicroRNAs are small non-coding RNAs inhibiting gene expression by pairing to the 3' UnTranslated Region (3'UTR) of their target mRNAs facilitating their translational repression/degradation. MiR-31 and miR-155 have been shown to be up-regulated in active UC and are involved in the regulation of innate and adaptive immune responses. Both microRNAs have been identified to target IL13R $\alpha 1$.

Methods Paired biopsies from inflamed and non-inflamed areas of the sigmoid colon were taken in 11 UC patients. MicroRNA expression and mRNA expression of IL-13 dependant genes were assessed by qPCR. In vitro experiments on a human colonic cell line (HT-29 cells) and a macrophage/monocytic cell line (THP-1 cells) were stimulated with IL-13. SOCS1 and IL13R $\alpha 1$ mRNA expression were measured by qPCR in the presence or absence of transfection with pre-miR-31, pre-miR-155 or a combination of both. Data were analysed using the Wilcoxon matched pairs test.

Results Our data shows a significant up-regulation of miR-31 and miR-155, as well as significant increase of IL-13 dependant mRNA expression of CCL18, SOCS1, Serpine and MMP-9 and a decrease of IL13R $\alpha 1 \mathrm{mRNA}$ expression by $30 \%$ in the active segments of UC as compared to inactive disease. In vitro experiments on HT-29 cells and on THP-1 cells showed a marked reduction of mRNA expression of SOCS1 and IL13R $\alpha 1$ in both cell types after treatment with pre-miR-31 and pre-miR-155 and their combination at a quarter of the original dose of each single pre-miR was equally efficacious.

Conclusion Our data reveals a clear up-regulation of miR-31 and miR-155 in inflamed UC as compared to neighbouring inactive tissue in the same patient. IL-13 dependant mRNA expression is also significantly increased in the same samples. The fact that IL13R $\alpha 1$ mRNA expression is down-regulated in active disease in the presence of high levels of miR-31 and miR-155 may indicate a protective role for these microRNAs attempting to reduce IL13R $\alpha 1$ expression and therefore the STAT6 pathway activation by IL-13 through IL13R $\alpha 1$. Reduction of IL13R $\alpha 1 \mathrm{mRNA}$ expression in our in vitro models transfected with pre-miRs confirms this finding. Interestingly the combination of the two microRNAs at lower doses achieving the same effect may indicate a possible synergy of action. MicroRNAs targeting IL13R $\alpha 1$ in UC could have a potential therapeutic effect by down-regulation of the IL-13/STAT6 pathway and combination of microRNAs may have a synergistic effect.

Competing interests None declared.

\section{OC-163 IDENTIFICATION OF INFLAMMATORY BOWEL DISEASE (IBD) USING FIELD ASYMIMETRIC ION MOBILITY SPECTROMETRY (FAIMS)}

doi:10.1136/gutjnl-2012-302514a.163

${ }^{1} \mathrm{R}$ Arasaradnam, ${ }^{2} \mathrm{~N} O$ Ouaret, ${ }^{2} \mathrm{M}$ Thomas, ${ }^{3} \mathrm{E}$ Hetherington, ${ }^{1} \mathrm{M} \mathrm{N}$ Ouraishi, ${ }^{*}$ ${ }^{1} \mathrm{C}$ Nwokolo, ${ }^{3} \mathrm{~K}$ D Bardhan, ${ }^{2} \mathrm{~J}$ Covington. ${ }^{1} \mathrm{UHCW}$, Coventry, UK; ${ }^{2}$ School of Engineering, University of Warwick, Coventry, UK; ${ }^{3}$ Rotherham NHS foundation trust, Rotherham, UK

Introduction Resident colonic bacteria, principally anaerobes and firmicutes, ferment undigested fibre. The resultant volatile organic compounds (VOCs) formed are dissolved in the faeces but also absorbed and excreted in the urine. We have previously shown that electronic nose (E-nose) analysis of urine VOCs distinguishes between Crohn's disease (CD), ulcerative colitis (UC) and healthy volunteers $(\mathrm{HV})$ : the underlying principle is pattern recognition of disease-specific "chemical fingerprint". High-Field Asymmetric
Waveform Ion Mobility Spectrometry (FAIMS) offers a possible alternative. The underlying principle is separation of VOC chemical components based on their different ion mobilties in high electric fields. We performed a pilot study in the above groups, the patients in remission (Rem) or with active disease (AD), to assess if this technology could achieve separation between the groups. The results were validated against E-nose analysis.

Methods 59 subjects were studied; HV $n=14$, UC (Rem) $n=18$, UC (AD) $n=4$; CD (Rem) $n=19, C D(A D) n=4$. Urine samples $(7 \mathrm{ml})$ in universal containers $(25 \mathrm{ml})$ were heated to $40 \pm 0.1 \mathrm{C}$. The headspace (the air above the sample) was then analysed using FAIMS The data were analysed by Fisher Discriminant Analysis.

Results The technique distinguished between the three groups. Additionally, patients with active disease could be distinguished from those in remission. These results were concordant with E-nose analysis.

Conclusion This pilot shows that urine VOCs, analysed by the different approaches of E-nose and FAIMS, the latter a novel application, can distinguish the healthy from those with UC and $\mathrm{CD}$ when disease is active or in remission. The two technologies together offer a non-invasive approach to diagnosis and follow-up in inflammatory bowel disease.

Competing interests None declared.

\section{OC-164 TREATMENT NAIVE ACTIVE ULCERATIVE COLITIS HAS A DISTINCTIVE MICRORNA PROFILE}

doi:10.1136/gutjnl-2012-302514a.164

${ }^{1,2} \mathrm{~A}$ Claridge, ${ }^{*}{ }^{1} \mathrm{~S}$ L Pender, ${ }^{1,2} \mathrm{M}$ Gwiggner, ${ }^{1} \mathrm{R}$ Morgan-Walsh, ${ }^{1} \mathrm{~T}$ Sanchez-Elsner, ${ }^{2} \mathrm{~J}$ R F Cummings. ${ }^{1}$ Department of Clinical and Experimental Sciences, University of Southampton, Southampton, UK; ${ }^{2}$ Department of Gastroenterology, University Hospital Southampton Foundation Trust, Southampton, UK

Introduction MicroRNAs (miRNAs) are becoming increasingly recognised as key players in a multitude of inflammatory pathways through their ability to regulate gene expression. Numerous miRNAs have been shown to have an abnormal expression in Ulcerative Colitis (UC), although published results are inconsistent possibly due to the grouping of different phenotypes and concomitant medication. Understanding the miRNA profile in homogeneous groupings is a major advance to understanding the mechanisms that underpin the aetiology of UC. The aim is to identify a distinctive miRNA signature for active sigmoid UC in patients who are treatment naive. Methods Sigmoid biopsies were taken from patients with Baron Grade 3 active UC $(n=21)$, patients with previous sigmoid UC who have no inflammation $(n=15)$ and healthy controls $(n=20)$. All patients were matched for age, sex, ethnicity and no medication for $\geq 6$ months. Deregulated miRNA candidates were first identified in active UC $(\mathrm{n}=6)$ and compared against controls $(\mathrm{n}=6)$ using Taqman ${ }^{\circledR}$ Array MicroRNA card A. Array cards were performed in pairs (active vs control). The differential expression of 377 miRNAs were calculated for each pair. The 20 miRNAs with the greatest increase and 20 with the greatest decrease in expression for each pair were then compared with the other array card pairs. MiRNAs that had a consistently increased or decreased expression in all array card pairs were validated using RT-qPCR. Differential expressions was calculated using the fold change compared with healthy controls. Statistical analysis was performed using Mann-Whitney test

Results Seven miRNAs were consistently deregulated across all miRNA array pairs. RT-qPCR confirmed a significantly increased fold change in three miRNAs; miR-31 (10.97, $\mathrm{p}<0.001)$, miR-223 $(3.45, p<0.001)$ and miR-146b-5p $(2.26, p=0.002)$. Four miRNAs were shown to have a statistically significant decreased expression by RT-qPCR. Only miR-31 and -223 were deregulated with statistical significance in inactive UC (fold increase of 2.83, $p<0.001$ and $2.47, \mathrm{p}<0.001$ respectively). 